\title{
Clinical Neisseria gonorrhoeae isolate with a $N$. meningitidis porA gene and no prolyliminopeptidase activity, Sweden, 2011 - danger of false-negative genetic and culture diagnostic results
}

D Golparian ${ }^{1}$, E Johansson ${ }^{1}$, M Unemo (magnus.unemo@orebroll.se) ${ }^{1}$

1. WHO Collaborating Centre for Gonorrhoea and other STIs, Swedish Reference Laboratory for Pathogenic Neisseria, Department of Laboratory Medicine, Microbiology, Örebro University Hospital, Örebro, Sweden

We describe a Neisseria gonorrhoeae strain, found in Sweden in 2011, that harbours a N. meningitidis porA gene causing false-negative results in PCRs targeting the gonococcal porA pseudogene. Furthermore, the strain had no prolyliminopeptidase (PIP) activity that many commercial biochemical kits for species verification in culture rely on. Enhanced awareness of the spread of such strains and screening for them can be crucial.

Gonorrhoea remains a global public health threat and the World Health Organization (WHO) estimated that 88 million new gonorrhoea cases occurred in 2005 [1]. In many laboratories worldwide, commercial or in-house nucleic acid amplification tests (NAATs) have rapidly replaced culture of the aetiological agent Neisseria gonorrhoeae for the diagnosis of gonorrhoea. The gonococcal porA pseudogene is possibly the most common target in in-house PCRs currently used for primary detection and/or verifying detection of $N$. gonorrhoeae globally. This is because the pseudogene is highly conserved and has so far been considered to be present in all gonococcal strains. It is also sufficiently diverse from the meningococcal porA gene, and commensal Neisseria species are lacking the porA gene/pseudogene [2-5]. However, recently the first case of a clinical N. gonorrhoeae isolate was found in Australia, in which the gonococcal porA pseudogene was replaced with a $N$. meningitidis porA gene sequence, which caused a false-negative result in a gonococcal porA pseudogene PCR [6].

This report describes the identification and detailed characterisation of the second case of a $N$. gonorrhoeae isolate harbouring a $N$. meningitidis porA gene that causes false-negative results in PCRs targeting the $N$. gonorrhoeae porA pseudogene.

\section{Case report}

In May 2011, a pharyngeal specimen from a woman in her 30 s presenting to a dermatovenerological clinic in Sweden was culture-positive for N. gonorrhoeae. The patient had recently had oral sex with a man in Sweden who could not be traced. She had no recent trips abroad. She was given therapy with cefixime (400 mg oral dose) and seven days later a test-of-cure using culture was negative, which indicated a successful treatment. However, it is known that culture, especially of pharyngeal specimens, has a suboptimal sensitivity compared to NAATs $[7,8]$.

\section{Characterisation of the N. gonorrhoeae} strain with a meningococcal por $A$ gene

The N. gonorrhoeae isolate was initially identified by typical colonies on selective culture medium, rapid oxidase production, presence of Gram-negative diplococci in microscopy, and two phenotypic species-verifying assays, i.e. an in-house sugar utilisation test and Phadebact GC Monoclonal Test (Bactus AB, Sweden).

When screening 200 clinical gonococcal isolates from 2011 with a PCR targeting the gonococcal porA pseudogene [2], the isolate obtained from the case above was repeatedly negative. Nevertheless, the phenotypic methods remained positive for $N$. gonorrhoeae, and additional phenotypic methods such as matrix-assisted laser desorption/ionisation time-of-flight mass spectrometry (MALDI-TOF-MS; bioMérieux, France) and API NH (bioMérieux, France) confirmed this species. However, the isolate did not show any prolyliminopeptidase (PIP) activity in the API NH. According to Etest (bioMérieux, Sweden), the isolate was susceptible to cefixime, ceftriaxone, ampicillin, ciprofloxacin and spectinomycin, but resistant to azithromycin (Table). The isolate was also identified as $N$. gonorrhoeae in 
APTIMA Combo 2 and APTIMA GC NAATs (Gen-Probe, United States).

For genetic characterisation, DNA was isolated in the robotised NorDiag Bullet (NorDiag ASA Company, Norway) using BUGS n'BEADS STI-fast kit (NorDiag ASA Company). The $16 \mathrm{~S}$ rRNA gene in the isolate showed $100 \%$ sequence identity with other N. gonorrhoeae strains in a GenBank BLAST search. The strain was assigned to $N$. gonorrhoeae multi-antigen sequencing typing (NG-MAST) ST2382 (porB allele 1480 and tbpB allele 4) and multilocus sequence typing (MLST) ST7367 (abcZ allele 109, adk 39, aroE 67, fumC 111, gdh 148, pdh (153, pgm 133), performed as previously described $[9,10]$. However, two gonococcal porA pseudogene PCRs $[2,4]$ gave negative results. Sequencing of the full-length gonococcal porA pseudogene, performed as previously described [3], identified instead a meningococcal porA gene sequence ( $94 \%$ sequence identity with the genome-sequenced meningococcal reference strain $\mathrm{MC}_{58}$ [11]), which was assigned to meningococcal genosubtype P1.21-6,2-48,35-1 (Table). This meningococcal porA sequence had multiple mismatches in the target sequences for both the primers and probe used in the two gonococcal porA pseudogene PCRs $[2,4]$. The monoclonal antibody $4 \mathrm{BG}_{4}-\mathrm{E} 7$ multivalent PorA (which is described and can be obtained at www.nibsc.ac.uk) verified that the meningococcal PorA protein was also expressed.

\section{Discussion}

There is one previously published report from Australia on a $N$. gonorrhoeae isolate that lacks the highly conserved gonococcal porA pseudogene [6]. We describe here the identification and characteristics of a N. gonorrhoeae isolate from Europe lacking the gonococcal porA pseudogene. The results from the present study together with the data from the Australian report [6] show that gonococcal strains can harbour a N. meningitidis porA sequence instead of the gonococcal porA pseudogene that causes false-negative results using N. gonorrhoeae porA pseudogene PCRs [2,4], which are commonly used in many laboratories globally. The isolate described in the present study also lacked PIP activity, which might challenge the species verification in culture if commercial biochemical kits such as API NH, RapID NH, Gonocheck II, Bacticard Neisseria and Neisseria Preformed Enzyme Test (PET) are used [12]. These kits are used worldwide and rely entirely or in part on the gonococcal PIP activity. This is of major concern, in particular because global transmission of PIP-negative gonococcal strains has previously been described [12]. The isolate described in the present study was assigned to MLST ST7367 (differing in two of the seven alleles from the previously described strain from Australia [6], i.e. which had $\operatorname{aroE} 170$ and pgm 65) and to NG-MAST ST2382 (differing from the previously described strain from Australia [6] by 65 bp in a sequence alignment of the por $B$ alleles and by $1 \mathrm{bp}$ in the $t b p B$ allele). Accordingly, this clone was not identical to the gonococcal clone reported from Australia, which was assigned to MLST ST1901 and NG-MAST ST5377 [6]. Thus it is clear that more than one gonococcal clone has acquired a meningococcal porA sequence, most likely through horizontal gene transfer and subsequent recombination.

It is worrying that the sexual contact of the present case could not be traced and this gonococcal strain could therefore be circulating in a larger sexual network. The findings of the present study have prompted us to carry out systematic screening of isolates from the past 10 years, which is currently ongoing.

In conclusion, the identification of a $N$. gonorrhoeae isolate harbouring a N. meningitidis porA gene as well as lacking PIP activity highlights the limitations and challenges using NAATs for diagnosis of gonorrhoea as well as in species verification in culture diagnostics for gonorrhoea. The presence of these two genetic changes in the same strain, which allow the strain to escape commonly used diagnostic tests, clearly illustrates how versatile the $N$. gonorrhoeae species is. Enhanced awareness of the spread of such strains is needed, and screening for them can be crucial. The opportunities to use combinations of different diagnostic methods (such as NAAT and culture) and multi-target NAATs in a laboratory remain exceedingly valuable.

\section{TABLE}

Characteristics of a Neisseria gonorrhoeae strain harbouring a N. meningitidis porA gene that causes false-negative results in gonococcal porA pseudogene PCRs, Sweden, 2011

\begin{tabular}{|c|c|c|c|c|c|c|c|c|c|c|}
\hline NG-MAST & MLST & $\begin{array}{c}\text { PIP } \\
\text { activity }^{a}\end{array}$ & $\begin{array}{l}\text { Ampicillin } \\
\text { MIC (mg/L) }\end{array}$ & $\begin{array}{l}\text { Ciprofloxacin } \\
\text { MIC (mg/L) }\end{array}$ & $\begin{array}{l}\text { Spectinomycin } \\
\text { MIC (mg/L) }\end{array}$ & $\begin{array}{l}\text { Ceftriaxone } \\
\text { MIC (mg/L) }\end{array}$ & $\begin{array}{l}\text { Cefixime } \\
\text { MIC (mg/L) }\end{array}$ & $\begin{array}{l}\text { Azithromycin } \\
\text { MIC (mg/L) }\end{array}$ & porA ${ }^{\mathrm{b}}$ & $\begin{array}{c}\text { porA } \\
\text { genosubtype }\end{array}$ \\
\hline $\mathrm{ST} 2382$ & ST7367 & Negative & 0.064 & $<0.002$ & 8 & 0.002 & $<0.016$ & 8 & $94 \% M C_{5} 8$ & $\begin{array}{l}\text { P1.21-6, } \\
2-48,35-1\end{array}$ \\
\hline
\end{tabular}

MIC: minimum inhibitory concentration (Etest was used); MLST: multilocus sequence typing; NG-MAST: Neisseria gonorrhoeae multi-antigen sequence typing; PIP: prolyliminopeptidase.

a The $N$. gonorrhoeae strain did not show any prolyliminopeptidase (PIP) activity, which might challenge the species-verification in culture if commercial biochemical kits are used that rely entirely or in part on the gonococcal PIP activity, such as API NH, RapID NH, Gonocheck II, Bacticard Neisseria and Neisseria Preformed Enzyme Test (PET). This is of particular concern because global transmission of PIP-negative gonococcal strains has previously been described [12].

b The porA gene in the $N$. gonorrhoeae strain showed $94 \%$ sequence identity with the porA gene in the genome-sequenced $N$. meningitidis reference strain $\mathrm{MC}_{5} 8$ [11] 


\section{References}

1. World Health Organization. Prevalence and incidence of selected sexually transmitted infections: Chlamydia trachomatis, Neisseria gonorrhoeae, syphilis and Trichomonas vaginalis. Methods and results used by WHO to generate 2005 estimates. Geneva: World Health Organization; 2011. ISBN:978 924150245 o. Available from: http://whqlibdoc.who.int/ publications/2011/9789241502450_eng.pdf

2. Hjelmevoll SO, Olsen ME, Sollid JU, Haaheim H, Unemo $M$, Skogen V. A fast real-time polymerase chain reaction method for sensitive and specific detection of the Neisseria gonorrhoeae porA pseudogene. J Mol Diagn. 2006;8(5):574-81.

3. Unemo M, Norlén 0 , Fredlund $H$. The porA pseudogene of Neisseria gonorrhoeae - low level of genetic polymorphism and a few, mainly identical, inactivating mutations. APMIS. 2005;113(6):410-9.

4. Whiley DM, Anderson TP, Barratt K, Beaman MH, Buda PJ, Carter M, et al. Evidence that the gonococcal porA pseudogene is present in a broad range of Neisseria gonorrhoeae strains; suitability as a diagnostic target. Pathology. 2006;38(5):445-8.

5. Whiley DM, Buda PJ, Bayliss J, Cover L, Bates J, Sloots TP. A new confirmatory Neisseria gonorrhoeae real-time PCR assay targeting the porA pseudogene. Eur J Clin Microbiol Infect Dis. 2004;23(9):705-10.

6. Whiley DM, Limnios A, Moon NJ, Gehrig N, Goire N, Hogan $T$, et al. False-negative results using Neisseria gonorrhoeae porA pseudogene PCR - a clinical gonococcal isolate with an N. meningitidis porA sequence, Australia, March 2011. Euro Surveill. 2011;16(21):pii:19874. Available from: http://www. eurosurveillance.org/ViewArticle.aspx?Articleld=19874

7. Mimiaga MJ, Helms DJ, Reisner SL, Grasso C, Bertrand T, Mosure DJ, et al. Gonococcal, chlamydia, and syphilis infection positivity among MSM attending a large primary care clinic, Boston, 2003 to 2004. Sex Transm Dis. 2009;36(8):507-11.

8. Schachter J, Moncada J, Liska S, Shayevich C, Klausner JD. Nucleic acid amplification tests in the diagnosis of chlamydial and gonococcal infections of the oropharynx and rectum in men who have sex with men. Sex Transm Dis. 2008;35(7):637-42.

9. Unemo M, Sjöstrand A, Akhras M, Gharizadeh B, Lindbäck E, Pourmand N, et al. Molecular characterization of Neisseria gonorrhoeae identifies transmission and resistance of one ciprofloxacin-resistant strain. APMIS. 2007;115(3):231-41.

10. Ohnishi M, Watanabe Y, Ono E, Takahashi C, Oya H, Kuroki T, et al. Spread of a chromosomal cefixime-resistant penA gene among different Neisseria gonorrhoeae lineages. Antimicrob Agents Chemother. 2010;54(3):1060-7.

11. Tettelin H, Saunders NJ, Heidelberg J, Jeffries AC, Nelson $\mathrm{KE}$, Eisen JA, et al. Complete genome sequence of Neisseria meningitidis serogroup B strain MC58. Science. 2000;287(5459):1809-15.

12. Unemo M, Palmer HM, Blackmore T, Herrera G, Fredlund H, Limnios A, et al. Global transmission of prolyliminopeptidasenegative Neisseria gonorrhoeae strains: implications for changes in diagnostic strategies. Sex Transm Infect. 2007;83(1):47-51. 